\title{
Erratum to: Regulation of arterial blood pressure by Akt1-dependent vascular relaxation
}

\author{
Jung Min Ha $\cdot$ Young Whan Kim • Dong Hyung Lee • \\ Sung Ji Yun • Eun Kyoung Kim • In Hye Jin • Ji Hyun Kim • \\ Chi Dae Kim • Hwa Kyoung Shin • Sun Sik Bae
}

Published online: 20 March 2013

(C) Springer-Verlag Berlin Heidelberg 2013

Erratum to: J Mol Med (Berl). 2011 Dec;89(12):1253-60

DOI 10.1007/s00109-011-0798-3

In Fig. 2a, reporting data from immunohistochemistry, the image of total eNOS staining in the NT condition was published incorrectly. Specifically, it was duplicated from the PDGF condition by mistake. In the corrected version of Fig. 2a, the image was replaced appropriately. The authors claim that this correction does not affect the message and conclusion of this study, and would like to apologize for this oversight.

The online version of the original article can be found at http://dx.doi.org/ 10.1007/s00109-011-0798-3.

J. M. Ha • Y. W. Kim· S. J. Yun • E. K. Kim• I. H. Jin •

C. D. Kim $\cdot$ S. S. Bae $(\bowtie)$

MRC for Ischemic Tissue Regeneration and Department

of Pharmacology, Pusan National University School of Medicine,

Bumeo-ri, Mulgeum-eup,

Yangsan-si, Kyungnam 626-870, Republic of Korea

e-mail: sunsik@pusan.ac.kr

D. H. Lee

Department of Obstetrics and Gynecology, Pusan National University School of Medicine, Bumeo-ri, Mulgeum-eup,

Yangsan-si, Kyungnam 626-870, Republic of Korea

J. H. Kim $\cdot$ H. K. Shin

Department of Anatomy, Pusan National University

School of Korean Medicine, Bumeo-ri, Mulgeum-eup,

Yangsan-si, Kyungnam 626-870, Republic of Korea 
Fig. 2 Phosphorylation of eNOS is abrogated in aortic tissues lacking Akt1. Aortic tissues isolated from mice were stimulated with PDGF $(50 \mathrm{ng} / \mathrm{ml})$ for $10 \mathrm{~min}$ and stained (a) or immunoblotted (b) with the indicated antibodies. Images were captured under confocal microscopy $(\times 40)$. c Aortic tissues isolated from wildtype $(W T)$ or Akt1 knockout $(1 K O)$ mice were lysed with lysis buffer, and the expression of each Akt isoform was verified by western blotting with the indicated antibodies. d Aortic tissues were stimulated with PDGF $(50 \mathrm{ng} / \mathrm{ml})$, and phosphorylation of eNOS was detected by immunohistochemistry using phospho-specific eNOS antibody (Ser1177). Images were captured under confocal microscopy $(\times 40)$. Staining of total eNOS was included as an internal control. Bars indicate $50 \mu \mathrm{m}$ a
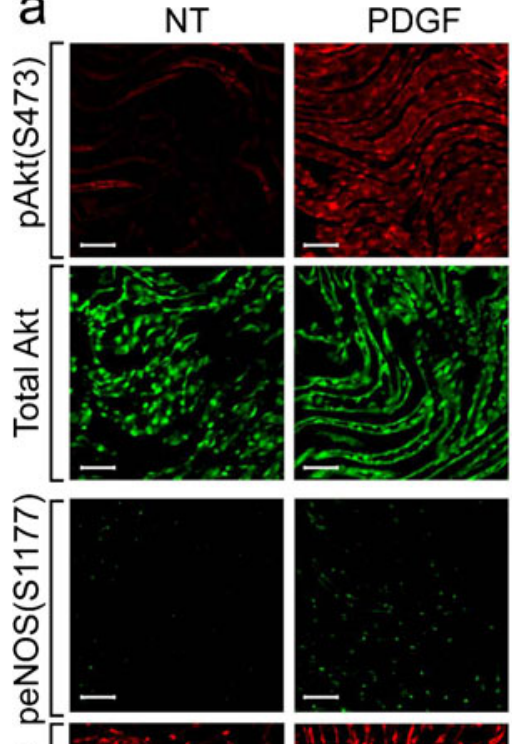

0)

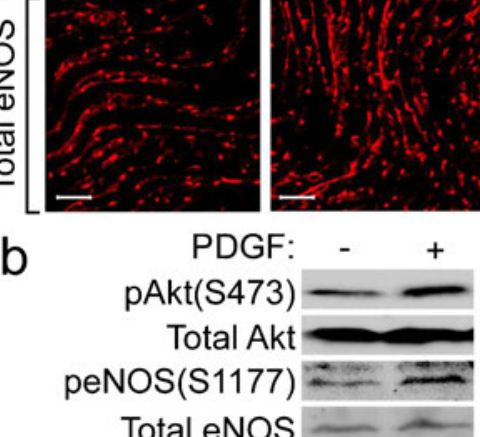

C

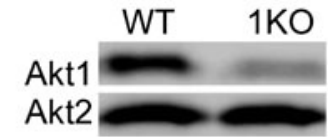

Total Akt

d
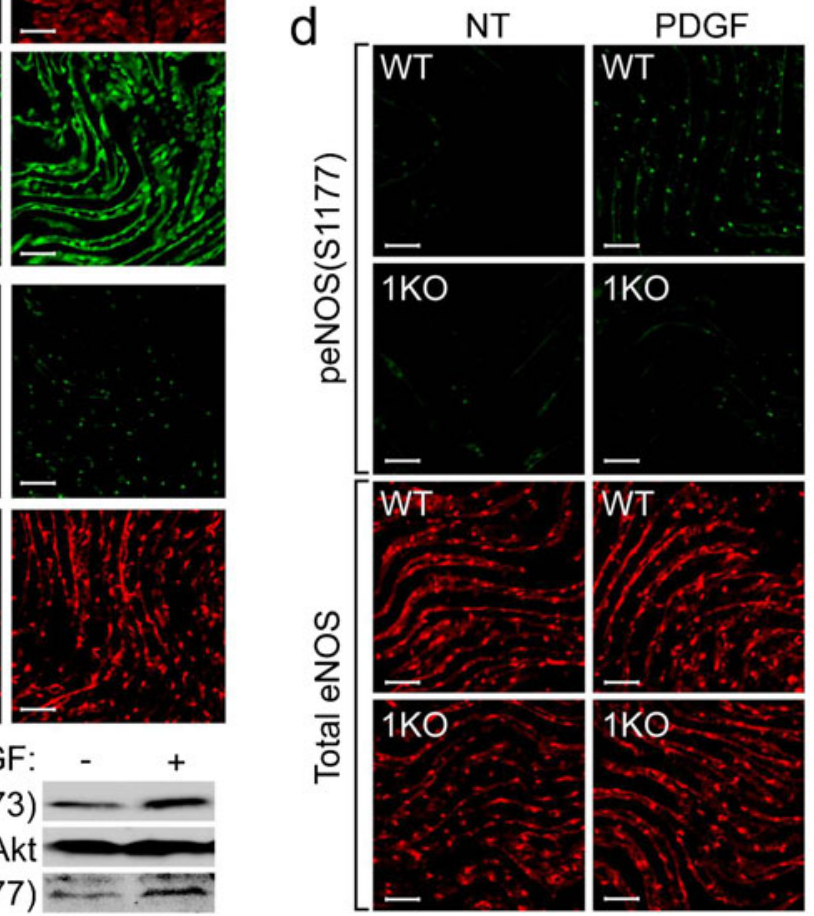